\title{
Lecture croisée du désir dans Hable con ella de Pedro Almodóvar et La Macération de Rachid Boudjedra à travers le motif de l'eau
}

\author{
M'selmi, Sana
}

Université La Sorbonne-Nouvelle Paris 3, $\underline{\text { Sana.mselmi@gmail.com }}$

\section{Resumen}

La elección de La Macération y de Hable con ella emana, entre otras cosas, de una similitud temática: el deseo de un hombre hacia una mujer que pasa o no por la palabra. La Macération y Hable con ella consiguen adelantar la porosidad entre literatura y cine a nivel poético (estético) para expresar la noción del deseo en su vínculo visceral con la imagen y la palabra acuática.

Palabras clave: deseo; agua; imagen; escritura mística; palabras.

\section{Résumé}

Le choix de La Macération et de Hable con ella découle, entre autres, d'une ressemblance thématique : le désir d'un homme pour une femme qui passe ou non par la parole. La Macération et Hable con ella réussissent à porter en avant la porosité entre littérature et cinéma au niveau poétique (esthétique) en vue d'exprimer la notion du désir dans son lien viscéral avec l'image et la parole aquatique.

Mots-clés: désir ; image ; élément aqueux ; écriture mystique ; paroles.

\begin{abstract}
The choice of La Macération and Hable con ella results from a thematic resemblance: the desire of a man for a woman which can be expressed or not by the word. La Macération and Hable con ella, seem to be the most capable to carry forward the porosity between literature and cinema at the poetic (esthetic) level to express the notion of desire in its visceral link with the aquatic image and discourse.
\end{abstract}

Keywords: desire; image; aquatic element; mystical writing; words. 
«Les poètes seuls devraient s'occuper des liquides », Novalis "Cher Marco, il pleut toujours je crois que c'est bon signe ${ }^{1}$

\section{Introduction : d'eau et de désir}

Dans Figures I, Gérard Genette parle de « chercher le sens et la cohérence d'une œuvre au niveau des sensations, des rêveries substantielles, des préférences avouées ou inavouées pour certains éléments, certaines matières, certains états du monde extérieur, au niveau de cette région de la conscience, profonde mais ouverte aux choses » (1966 : 91). Dans le même ordre d'idées, Bachelard, dans L'Eau et les rêves, affirme: "Seule une matière peut recevoir la charge des impressions et des sentiments multiples. Elle est un bien sentimental » (1942 : 238). A cet égard, le développement de l'imaginaire de l'eau dans une œuvre donnée ne peut qu'être éloquent et symbolique et ce, à plus d'un titre.

Dans certains textes védiques, la présence de l'eau précède la création du monde, le commencement de l'univers se résumait à une onde. Selon le Dictionnaire des mythes littéraires, l'eau exprime le désir de se reproduire (Brunel, 1988 : 366-367). Certains poètes et auteurs dotent la mer et par conséquent l'eau de sexualité ; Rudolf Kjellén parle, dans ce sens, du motif de sexualité aquatique. Il est à noter, en outre, qu'une des constantes de la description en littérature est la métaphore qui consiste à rapprocher le désir érotique et le motif du torrent. L'association de la pluie et du désir fait aussi partie d'une longue tradition littéraire perpétuée entre autres par George Sand, Marguerite Duras, etc. Le septième art n'est pas en reste, les célèbres scènes où l'eau qui coule exacerbe les désirs sont nombreuses. Je cite par exemple Tant qu'il y aura des hommes (Zinnemann, 1953), Vertigo (Hitchcock, 1958), ou même le plus récent In the Mood for Love (Kar-way, 2000).

La littérature, selon Marc Gontard (2006 : 1), aborde sous une perspective à chaque fois renouvelée une question unique et universelle : la question du désir. La littérature maghrébine, en particulier, a toujours fait du désir une constante qui travaille le texte en profondeur et le métamorphose. Opérateur de la narrativité, point d'ancrage et de départ du processus scriptural, le désir est protéiforme. Transitif toujours, compulsif souvent, aliéné et aliénant, il interpelle, dans la lecture de l'œuvre de l'Algérien Rachid Boudjedra, et notamment dans le roman qui m’intéresse ici La Macération (1984). Cette présence du désir est tellement forte que certains critiques parlent de l'écriture « sexuelle » ou « érotique » de Boudjedra². La particularité de ce romancier, c'est d'avoir su marier l'érotisme -souvent considéré comme un domaine littéraire tabou ou, si toléré, de seconde catégorie-à une écriture innovante et novatrice, qui a pu transmuter son œuvre vers une esthétique moderne et universelle. Mais comment se manifeste l'érotique au niveau du texte ? Quel rôle joue l'eau dans l'expression du désir?

Pedro Almodóvar, d'un autre côté, est sans conteste le cinéaste du désir par excellence, en témoigne les titres de ses films ; de La Ley del deseo (La Loi du désir) (1987), à Carne tremula (En chair et en os) (1997), du Laberinto de pasiones (Labyrinthe des passions) (1982) à i Átame ! (Attache-moi !) (1989), la filmographie du cinéaste manchego ouvre grand la porte à l'Eros qui s'y manifeste de bien de manières. Amours tragiques, passions déchirées et déchirantes, corps en perpétuelle tourmente aux prises avec le désir d'être autres, l’univers almodovarien est fait de films «[d]érangeants, outranciers, provocateurs, d’un érotisme exacerbé, [...] conduis[a]nt dans des sphères narratives inexplorées par le cinéma non pornographique. » (Castel, 2013 : 41-42). Dans le cinéma de Pedro Almodóvar, Eros et Thanatos sont intimement liés. A chaque plan, à chaque séquence. L'univers de Pedro Almodóvar est certes citadin mais il est loin d'être aride. Il est riche de par sa palette chatoyante et par la métaphore de l'eau qu'il file tout au long de sa filmographie. En effet, Hable con ella (2002) n'est pas le premier film à donner à l'eau un rôle diégétique capital. Il suffit de se rappeler de la pluie de la nuit fatidique où Esteban est mort dans Todo sobre mi madre (1999), de la scène de bain de i Átame !, mais

\footnotetext{
${ }^{1}$ Hable con ella, Benigno en maison d’arrêt s’adressant à son ami Marco.

${ }^{2}$ Ce dernier a de tout temps été taxé d’être un écrivain subversif. Et dès la parution de son premier roman (La Répudiation), il est considéré comme « l'enfant terrible » de la littérature maghrébine. Mais il est à noter que l'écrivain algérien hérite d’une longue tradition de littérature transgressive. Des poètes arabes préislamiques - qu'il cite souvent dans son œuvre -, ainsi que des écrivains considérés comme subversifs en terre d’Islam comme Nefzawi et son jardin parfumé. La Prairie parfumée, (ou Le jardin parfumé) de son nom complet La Prairie parfumée où s'ébattent les plaisirs (الروض العاطر في نز هة الخاطر), est un ouvrage de littérature érotique arabe écrit par Cheikh Nefzaoui et traitant de la sexualité dans ses multiples facettes. Il aurait été rédigé à la demande de Abû Fâris `Abd al-`Azîz al-Mutawakkil, souverain hafside de Tunis. Selon l'introduction de la traduction anglaise de Jim Colville, Nefzaoui aurait probablement rédigé le texte entre 1410 et 1434.
} 
surtout de cette scène mythique où le transsexuel Tina se fait arroser par le tuyau de l'agent de voieries madrilènes, un soir de canicule dans La ley del deseo.

Dans cet article, je me propose d'illustrer l'importance de l'eau dans La Macération et Hable con ella. L'eau, en effet par sa présence comme par son absence-, donne à l'œuvre « sa propre substance, sa poétique spécifique ». Il s’agit ici d'étudier la dichotomie des images aqueuses de l'Eros (flux et reflux ; positif et négatif ; féminin et masculin ; mais surtout parole et mutisme) dans Hable con ella, et de démontrer comment La Macération, comme son titre l'indique, est un long roman dont le processus scriptural met en pratique le principe de la macération : humidité et immobilisme diégétique, stagnation textuelle et métaphore aquatique au service d'un Eros triomphant.

\section{Les mots liquides, ferments du désir}

Généralement, le désir se traduit en actes. Actes manqués ou actes aboutis. Mais dans l’univers de La Macération et dans Hable con ella, le désir est avant tout paroles. Alors que dans le film les mots tendent à véhiculer le désir sans espoir de réponse, les mots dans le roman sont les ferments du désir ; la parole y est nettement aphrodisiaque. Les mots se saisissent du désir et le fragmentent en syllabes et lettres brûlantes :

[...] toutes ces couleurs, impressions, sensations - donnent l'idée d'un écoulement aquatique ou plutôt d'une source souterraine sinuant sous une surface gelée et gercée, mais qui - essentiellement - joue le rôle d'un ferment qui fait lever et gonfler les phénomènes que l’on vit au plus profond des couches du sommeil, phénomènes qui fermentent sous l'effet de certains mots obsessionnels et lourds de sens ; de mouvements étranges et désarticulés ; de paysages détramés, brouillés et rayés ; de souvenirs démantelés, liquéfiés, effilochés ; ce qui fait que leur signification s'entête à demeurer obscure, flottant à la surface des eaux troubles et stagnantes, à tel point qu'ils macèrent (les mots), pourrissent, moisissent et se corrompent au point de former un champ marécageux où les lettres (Sache donc que la relation qui régit certaines lettres est de l'ordre du désir et de la passion amoureuse! Ibn Arabi, Les Conquêtes mecquoises. Premier voyage : 260) ${ }^{3}$ ne tardent pas à se détériorer, à s'effriter et à surir ; de telle sorte que je ressentais -à chaque réveil laborieux- que mon corps s’était rétréci, hérissé et comme lézardé sous l'effet des phantasmes et des obsessions ; voire fissuré par l'inoculation de tant de sensations troubles et ambiguës [...] (Boudjedra, 1984 : 8).

Dans le roman donc, les mots agissent comme un levain. Un levier au service du moi souterrain qui se raconte à travers la métaphore de l'eau : eau qui coule à flot et eau qui stagne. Mais les mots se divisent, se multiplient, procréent et donnent naissance à d'autres mots. Le lecteur perd le fil de sa lecture et le narrateur macère dans le récit de ses peurs, angoisses, hésitations et tourmentes quotidiennes. Cet aperçu détaillé d’une détérioration des mots par macération est entrecoupée par une injonction puisée chez Ibn Arabi ${ }^{4}$, où il est question d'amour, de désir et de passion. Les lettres, les mots par conséquent, jouissent d'une âme (passion) et d'un corps (désir). Les mots sont donc le fruit d'une rencontre entre lettres, lettres qui ont répondu présentes à l'appel de la chair. La macération transforme les mots et liquéfie les corps et les sentiments. Le maître des mots est à la fois le sujet et l’objet du processus de macération.

Le roman met en scène la rencontre mouvementée entre un écrivain Rachid et son amante Maria-Myriam. Cette dernière le poursuit jusque dans sa retraite dans la maison familiale ; elle traque ses secrets et ses pensées intimes. Elle le pousse dans ses retranchements avec des questions intrusives, et de ce fait, déroule le récit de la mémoire qui s’enchevêtre avec celui de la relation brûlante qui unit les deux personnages. Maria, et le lecteur avec elle, assistent aussi au spectacle de l'écrivain tout à son acte créateur. La posture scripturale met, de ce fait, en scène, à la fois le voyeurisme et

\footnotetext{
${ }^{3}$ Les références sont ainsi citées dans le texte de Boudjedra qui souligne en utilisant les italiques. Il est à noter que les extraits puisés chez Ibn Arabi, le sont par l’auteur, ce qui implique que les références (numéros de page et autres) sont relevées par lui. Nous nous contentons de les citer comme elles paraissent dans La Macération.

${ }^{4}$ Moheïddine Ibn 'Arabi, espagnol musulman, d'origine arabe, plus connu sous son seul nom de Ibn Arabi (né le 7 août 1165, à Murcie, en al-Andalûs (actuelle Espagne), et mort le 16 novembre 1240, à Damas en Syrie). Également appelé « ach-Cheikh al-Akbar » (« le plus grand maître », en arabe), ou encore « Ibn Aflatûn » (le fils de Platon), il est un théologien, juriste, poète, métaphysicien et maître arabe-andalou du taçawuff islamique, auteur de 846 ouvrages. Son œuvre domine la spiritualité islamique depuis le XIIIe siècle, et il peut être considéré comme le pivot de la pensée métaphysique de l'Islam. Il est le plus grand penseur de la doctrine ésotérique du « wahdat al wujud ». Il eut quelques ennemis dans le domaine exotérique. Dans l'ésotérisme islamique, il est considéré comme le « sceau de la Sainteté ». Certains considèrent que son œuvre aurait influencé Dante.
} 
l'exhibitionnisme, où l'écriture agit sur l'amante-voyeur comme un aphrodisiaque. Cette posture est, donc, le prétexte à un déchaînement érotique, une union entre l'écrivant et l'amante, faisant défiler l'image de l'eau stagnante :

[...] lorsque je te vois écrire, l’odeur des marécages herbeux remplit mes narines [...] et m'emporte dans une sorte d'extase non pas mystique (je lui avais parlé un jour d’Ibn Arabi un mystique qui avait divisé les lettres en deux : les amoureuses et les non-amoureuses) mais sexuelle. J'écrivais donc pendant qu'elle restait derrière mon dos, des heures durant, surveillant la main devenue une sorte de tenaille mécanique qui glisse furtivement sur un rythme ininterrompu, liée à ce fil conducteur qui se déroule dans la tête de l'écrivant et enroule tant de choses et de visions qui fermentent, rancissent, aigrissent et -surtout- macèrent longuement dans le liquide de la vie [...] (Boudjedra, 1984 : 92-95).

En développant la dyade parole-désir, le texte, contrairement au film, fait coïncider parole érotico-aquatique et acte désirant. Les mots se déploient au moment de la rencontre des corps. Ils accompagnent les personnages jusqu’à l'extase.

Le désir est souvent défini comme étant un vide qui cherche à devenir plein. C’est une quête frénétique de l'Autre. Le désir cherche l'union, l'osmose avec l'Autre. L'être désirant cherche à conquérir l'être désiré, à l'atteindre, le posséder, l'absorber. Dans cette perspective, Benigno a souvent recours à ses mots pour venir à bout du vide créé par l'absence de l'aimée (plongée dans le coma). Le narrateur de La Macération de son côté, tel une Schéhérazade au masculin, tisse autour de l'amante une toile de mots et d'images. Commence, alors, la ronde des mots, une ronde qui semble se déployer pour ne plus prendre fin. Les conversations-confessions sont à l'image de la rencontre des corps : une lutte impitoyable, un échange de mots-coups de poing ; de mots-morsures ; de mots-griffures du côté du roman et un soliloque sans fin du côté du film.

Dans La Macération, de stimulateur du désir, écrire devient petit à petit un acte sexuel. L’écriture peut être considérée comme faisant partie de l'acte d'amour. Elle constitue comme une étape, des préliminaires en quelque sorte. Le texte va plus loin en faisant du sexe féminin un encrier, du calame -ou crayon- l'équivalent, plus que la métaphore, du sexe mâle. L'encre se confond avec l'eau du désir et de vie. A ce propos, je me permets de citer le texte malgré la longueur de l'extrait :

Je sortis mon index de son trou. Je pris un crayon. Je le fis entrer dedans. Je sentis qu'il tournait sur lui-même une fois et une autre fois. Il pivotait autour d'un axe imaginaire qui serait sa colonne vertébrale. Elle commença à haleter. L'anesthésie la prenait de plein fouet et arrivait de très loin. [...] Je sentis son eau monter en elle le long de l'axe du désir. Elle coula fil à fil, visqueuse, épaisse, lourde, collante. J'augmentais la vitesse de mon mouvement. Elle murmura : l'écriture... L'écriture... Ecris-moi... Fais de moi une feuille de papier sur laquelle tu traces tes signes, tes lettres. [...] Langue (la languette de son sexe jaillit brusquement ... gonflée par le plaisir qui remontait à travers les hanches, les cuisses, vers la tête où elle se répandait dans les coins et les recoins de toutes les circonvolutions, jusqu'à atteindre le faîte du mûrier où les oiseaux ...) Elle avait dépassé le sentiment de pudeur ... J'allais de plus en plus vite, en tournant le crayon dans son vagin qui donnait l'impression de s'évaser et de s'évider ... elle balbutia : la sève... l'encre végétale... l'encre violette... Ce mouvement qui s'amplifiait touchait de plein fouet cette passion dont j'étais victime. Son eau phréatique coulait comme si elle sortait du vieil encrier en cristal de Bohême. [...] Aucune différence. Une manière d'écrire, de fabriquer de l'écriture en tant que matière brute. Le reste (le sens) ne doit être que secondaire ! Une manière de tourner la pointe du crayon (C'est ainsi qu'on peut définir le rapport existant entre la plume et le papier comme un rapport sexuel ; et qu'on peut appeler semence l'encre dont s'imprègne le papier et qui le féconde à la manière de la semence mâle qui inonde les entrailles de la femelle. Ibn Arabi, Les Conquêtes mecquoises. Deuxième voyage, p. 314 $)^{5}$ dans la chair des mots... Son corps aussi était une matière brute. Je faisais tourner le crayon de plus en plus vite. L’écoulement devint diluvien. Sa crue déferlait sur mes mains, les inondait : un tissu de fils lourds comme

\footnotetext{
${ }^{5}$ Dans la chronique « Empreinte Digitale », sous le titre « La littérature et le sacré », parue in Revue africaine le 15 avril 1988, Boudjedra évoque le même texte d’Ibn Arabi cité ici-même dans La Macération, avec ceci en plus : «En outre ce qui apparaît sur le papier comme écriture (ou littérature, c’est-à-dire ensemble de mots ou leur rassemblement, signifiants à travers les lettres), peut être aisément comparé aux âmes des enfants déposés dans leurs corps. " [Ibn Arabi, Les Conquêtes mecquoises, Deuxième voyage, p.314, El-Farabi]. Cité par Mohammed-Salah Zeliche, L'écriture de Rachid Boudjedra. Poét(h)ique des deux rives, Paris, Karthala, 2005, 353 pages, p.231.

Ainsi donc, dans la vision soufie, les lettres ont entre elles des rapports de l'ordre du désir charnel. De plus le lien entre la plume ou le calame et le papier est aussi de l'ordre de l'Eros. Les lettres, les mots et les phrases sont, de la sorte, le résultat de cette union entre calame-pénis et papier-sexe féminin. Les mots sont des enfants de l'amour. Dans ce sens, la littérature toute entière est à la fois un geste et un acte d'amour mais aussi l'objet qui naît de cet amour charnel.
} 
du mercure concentré. Elle commença à s'affaisser. Les choses s'effritèrent à l'exception du vase en cristal de Bohême et de l'encrier [...] Les choses s'étaient brouillées dans mon esprit. Je finis par sortir le crayon, dégoulinant de son eau, dégouttant, imprégné. Je pris une feuille vierge et j’écrivis avec sa propre matière liquide ( «je t’aime »). Je ressentis à ce moment-là une sorte de concentration unique en son genre. Fraîche et fragile : ces sept lettres incisées... Sa chair était tendre, molle, brûlante. Une chair brute comme désossée (Boudjedra, 1984 : 228-229).

Les mots semblent être le moyen qui assure la métamorphose du désir, de désir charnel à la passion mystique, en passant par l'acte sexuel. Ceci se fait à travers le déploiement de toute une isotopie de l'eau (eau, coula, visqueuse, épaisse, lourde, eau phréatique, écoulement diluvien, inondait, dégoulinant, dégouttant, matière liquide, etc.). L’amante interpelle Rachid : «écris-moi ! ». De la sorte, elle se fait papier qui appelle à être rempli de signes et de mots. Elle est poème. L'érotisme, à travers les mots du désir, atteint au mystique, au transcendantal. Le texte en appelle à Ibn Arabi pour donner de la puissance à cette fusion entre chair et langue, entre l'érotique et le divin. L'auteur, dans La Macération, ne déclare pas sa flamme à Maria-Myriam avec un discours ordinaire, il grave sa déclaration sur une feuille avec sa semence mélangée aux « liquides intimes » de l'amante ${ }^{6}$. L'acte sexuel ne fait pas que procéder de la parole (langue), il crée la parole. L'acte sexuel est un acte linguistique, un acte scriptural (et scripturaire) qui met en scène l'imaginaire de l'eau en ce qu'il est le reflet des sentiments, et de la rencontre amoureuse et érotique. L'eau figure l’Eros en fête :

Ils se turent en même temps. Se complétèrent. Il prit la feuille sur laquelle il avait écrit Je t’aime avec son eau souterraine, alors que les lettres commençaient à s'effacer. De l'eau glaireuse sur le papier fin. Il se dit où se trouve donc la frontière entre elle et moi (Boudjedra, 1984 : 275).

Et où se trouve la frontière entre sexe et texte, entre désir et écrire ? Selon Gaston Bachelard, l'eau est le composant des forces imaginantes particulièrement manifestes dans les œuvres littéraires, puisqu’elle est « la métamorphose ontologique essentielle. L'être voué à l'eau est un être en vertige » (1942 : 13). C'est le cas des amants de Boudjedra et c'est aussi le cas des personnages d'Almodóvar même si l'ivresse du désir dans le roman ne peut se confondre avec le vertige de la névrose causée par le désir non abouti dans le film.

\section{La parole torrentielle et stérile}

Le rapport de la parole avec le désir est autrement plus complexe dans le film espagnol. Alors que dans le roman, les mots porteurs d'une symbolique aqueuse sont l'adjuvent du désir, dans le film, ils sont incapables de véhiculer cet élan. Les longues confessions de Benigno ressemblent, certes, à un flux diluvien, mais ils ne font qu'étouffer le désir sous leur avalanche. Ici, la parole est aquatique de par son aspect torrentiel et non pas par les images érotiques qu'elle porte. Et ceci justement parce qu'il y a rarement d'échange dans le film, il y a surtout des conversations à sens unique. La réplique de Marco, excédé, s'adressant à un Benigno plein d'illusions est à cet égard très éloquente : « Ton truc avec Alicia c'est un monologue ».

Ainsi, plus qu'un film sur la parole -malgré la thèse du titre- Hable con ella est, donc, un film qui glisse vers la monstration de l'importance du regard dans la création et le jaillissement de l'émotion. La parole désirante est vaine, c'est l’image qui se charge du dire désirant à travers notamment le recours au film muet et aux spectacles dansants.

A propos de l'ampleur donnée à la parole et à son opulence dans ce film d'Almodóvar, je dirais que dans son œuvre de façon générale et ici en particulier, quand le verbe existe, se développe, enfle il semble faire de l'ombre à l'acte désirant. C’est dire que ce n'est pas vraiment le verbe qui véhicule le désir ; que le désir n’a pas besoin du verbe pour s'épanouir, ou du moins, pour survivre. Dans le film, et contrairement au roman, la parole ne coïncide presque jamais avec l'acte, leur coexistence semble impossible à réaliser. D’ailleurs, la réplique clef dans ce film est celle de Lydia à Marco : «On va parler après la corrida ». Parole qui se fait attendre et qui ne parvient jamais.

\footnotetext{
${ }^{6}$ Bachelard, à ce sujet, aborde les eaux maternelles et féminines : l'eau abreuve et nourrit le petit d'homme et le poète.
} 
Pour schématiser un peu : nous avons un film construit sur une structure quadrilatérale. A priori, nous avons deux couples, Lydia et Marco d'un côté, et Benigno et Alicia de l'autre :

- $\quad$ Lydia est un être désirant, elle est porteuse de la promesse de parole et du désir de parole inassouvi. Face à elle, il y a Marco, un être non désirant. Il choisit le silence, il retient ses émotions.

- Alicia est un être non désirant aussi. De par son état végétatif, elle est un être de silence. Chez elle, il n’y a pas de désir. En revanche, Benigno est non seulement un être désirant, mais son désir s'exprime et s'accompagne par une pléthore de paroles.

Ce schéma montre une déstructuration, une symétrie bancale :

- Marco se tait face au désir de parole de Lydia qui meurt en emportant avec elle sa parole, comme si le silence de Marco avait contribué à tuer sa maîtresse et son désir avec.

- Le silence d'Alicia face à la profusion parlante de Benigno a enfermé ce dernier dans la folie, puis l'a conduit à la mort.

Ceci induit à comprendre que :

- $\quad$ Dans le cas de Lydia : le désir de parole tue.

- Dans le cas de Benigno : le verbe diarrhéique tue.

- Dans le cas de Marco : le silence préserve.

- Dans le cas d'Alicia : le silence la préserve. Et ce sont le regard désirant et surtout les larmes finales de Marco qui les sauvent tous les deux. Le désir sans expression verbale se cherche et aboutit sur l'assouvissement.

Lorsque le verbe est, le désir n'est pas satisfait. Lorsque le silence est, le désir s’assouvit. Il semblerait donc que le désir échoue quand son véhiculaire est le verbe ${ }^{7}$. La parole, donc, n’est pas forcément le vecteur du désir. Au contraire, les émotions -dans ce film du moins- semblent s'épanouir quand elles se libèrent du joug du verbe. Prenons l'exemple du spectacle Café Müller de Pina Bausch qui suscite les larmes de Marco, Marco qui attire l'attention de Benigno et qui, par ses larmes parvient jusqu'à Alicia dans son état végétatif. Pina et Marco semblent conquérir l'empathie et la sympathie du spectateur et l'adhésion du réalisateur. Le motif aqueux à travers les larmes joue un rôle de fédérateur, de vecteur d'émotions et de désirs. Par ailleurs vers la fin du film, ce sont les larmes qui, encore une fois, unissent Marco et Benigno dans la maison d'arrêt où, par un effet de gros plan réfléchi sur la vitre qui sépare le prisonnier de son ami, leurs deux visages larmoyants se superposent. Cette image presque liquide avec la pluie en arrière-plan, la transparence du verre et le flou des larmes permet la transmission des émotions de Benigno vers Marco. Il se passe comme une sorte de circulation du désir d'Alicia, de Benigno vers son ami. Une sorte de passation des désirs, de testament émotionnel qui n’a pu avoir lieu que grâce au partage des larmes.

\section{De la faillite du verbe liquide au triomphe du féminin et de l'imago bucolique}

D’après Bachelard, le motif de l'eau illustre « le concept de bipolarité ». L'eau, en effet, est la matière qui exprime le plus l'ambivalence des sentiments et des émotions. De fait, la relation entre Rachid et Maria oscille entre amour et haine, fascination et répulsion. Les personnages almodovariens sont tout aussi partagés. Benigno est plus féminin que masculin et Marco aussi. Les images aquatiques sont dichotomiques: ou bien positives ou bien négatives; et ainsi elles infléchissent la nature du désir : par exemple, la pluie dans Hable con ella joue le double rôle d'obstacle et d'adjuvent à l'élan qui porte Benigno vers Alicia. Elle provoque l'accident de cette dernière et donc l'introduit dans l'univers de l'infirmier -même en tant que patiente-, et elle accompagne les derniers jours de Benigno dans la maison d'arrêt. La pluie qui tombe, à ce moment-là, isole encore plus Benigno dans sa névrose, sa solitude et son amour obsessionnel.

L’image que suggère l'eau est « une image fuyante, une image en fuite, car l'élément qui la porte et la constitue est voué par essence à l'évanouissement» (Genette, 1966 : 28). L'eau est donc par essence insaisissable, elle ne peut être circonscrite et reste mystérieuse, «elle est, selon Bachelard, un élément plus féminin [...] que le feu ». Et le film d'Almodóvar ne cesse de le montrer à travers les larmes qui coulent souvent d'yeux virils. L'eau est souvent considérée

\footnotetext{
${ }^{7}$ Pour rappel : Esteban, fils de Manuela dans Tout sur ma mère, meurt au moment où il allait obtenir sa réponse à propos de l'identité de son père, au moment où il allait avoir accès au verbe.
} 
comme la métaphore du féminin qui accède à la force, et de ce fait, les larmes de Marco peuvent être vue comme l'émergence de son moi féminin profondément enfoui en lui. Cette hypothèse corrobore celle de l’homosexualité refoulée, et pour l'expliquer, il me faudrait revenir à une scène culte de la filmographie almodovarienne, celle de la baignoire dans i Átame !. Dans cette scène, Marina prenant son bain, joue avec un plongeur miniature qui s’obstine à se nicher entre ses jambes. Ce mini-plongeur, on le retrouve dans Hable con ella à travers le nageur nocturne et Alfredo dans le métafilm Amante Menguante (L'Amant qui rétrécit). Le plongeur de la piscine grandeur nature mais filmé dans un cadrage large ce qui le rapetisse un peu- et qui squatte le rêve-flash-back de Marco rappelle cet autre plongeur dans une baignoire en guise de piscine. Alfredo, devenu lui-même homme-miniature qui s’introduit définitivement dans le vagin de son amante Amparo («refuge » en espagnol), ainsi que cet autre nageur constituent une sorte d'auto-plagiat qui permet à Pedro Almodóvar d’introduire l'image du corps masculin dans l'inconscient de Marco et de donner au spectateur plusieurs pistes d’interprétation concernant l'amitié qui le relie à Benigno.

L’immersion dans l'eau est régénératrice, elle opère une renaissance et établit l’être dans un état nouveau. Ainsi, l'image d'Alfredo plongeant dans le vagin d'Amparo se lit comme une plongée-retour aux sources originelles à savoir la féminité. Et d'ailleurs, le film en entier est à regarder comme un hommage à la femme, à l'unité sexuelle et à l'espoir bourgeonnant grâce à l'imaginaire de l'eau, imaginaire déployé dans Masurca Fogo par exemple. Ce spectacle dansant signé Pina Bausch exprime via une image bucolique et florale l'espoir, la sensualité, l'érotisme et la puissance du corps féminin qui s'affirme. Outre la cascade qui coule sur scène, l'image aquatique est exprimée à travers la gestuelle des danseurs portant à bout de bras une femme alanguie. « Comme des vagues, comme des vagues cruelles le masculin tout en bas et le féminin tout en haut », selon la description de Katerina, la maîtresse de ballet. L’eau coule sur scène donc et Marco retrouve le sourire et peut-être même un début de chemin vers le cœur d'Alicia, émue par ses larmes comme l'a été Benigno, avant elle, au tout début du film, devant un autre spectacle de Pina.

Cette fin ouverte sur l'espoir me rappelle une remarque faite par Jean-Claude Vareille à propos de George Sand : « Sand nous apprend qu'il faut franchir l'(eau) pour accéder à la réalisation érotique ou sociale. En traversant l'(eau), on trouve l'amour, l'utopie - et aussi, bien sûr, ultime volet d'une triade [...] l'art ou la beauté » (Vareille, 1983 : 129). Traverser l'eau est donc une sorte d’épreuve, un parcours initiatique que seuls quelques privilégiés sont capables de réussir.

\section{Conclusion}

Il est plausible, enfin, de parler d’une signature aquatique de l'écrivain algérien dans La Macération et de Pedro Almodóvar dans Hable con ella, puisque le premier relie l'imaginaire de l'eau à l'écriture érotico-mystique, et le second fait de l'eau une métaphore de la féminité triomphante.

Le romancier met au profit de son écriture le principe de liquéfaction afin de produire un texte où l'érotique se confond avec le mystique dans une fluidité exemplaire. Le cinéaste de son côté, recourt à l'esthétique liquide pour faire ressortir l'unité charnelle, l'osmose amoureuse et le jaillissement de l'émotion. L'imaginaire de l'eau travaille la poétique de l'un et de l'autre dans leur expression du désir.

\section{Références bibliographiques}

Almodóvar, Pedro (2002). Hable con ella. España.

BACHELARD, Gaston (1942). L'Eau et les rêves. Paris : José Corti.

Bausch, Pina (1978). Café Müller. Allemagne.

Bausch, Pina (1998). Masurca Fogo. Allemagne.

BoudJEDRA, Rachid (1984). La Macération. Paris : Denoël.

BRunel, Pierre (1988). Dictionnaire des mythes littéraires. Paris : Editions du Rocher.

CASTEL, Elie (2013). «Survol méditerranéen » dans 24 images, n³6. 41-42. <http://id.erudit.org/iderudit/22179ac> [Consulté le 19 septembre 2013].

GeNETTE, Gérard (1966). Figures I. Paris : Seuil. 
GonTARD, Marc (2006). «L’écriture du désir chez J.R. Léveillé. Entre turbulence et planéité ». <https://halshs.archivesouvertes.fr/halshs-00009346> [Consulté le 15 septembre 2014].

Нiтснсоск, Alfred (1958). Vertigo. États-Unis.

KAR-WAY, Wong (2000). In the Mood for Love. Hong Kong.

VAREILle, Jean-Claude (1983). «Fantasmes de la fiction. Fantasmes de l'écriture » dans Simone Vierne (dir.) George Sand, Colloque de Cerisy. Paris : Ed. C.D.U. Sedes. 129-138.

ZinnEmann, Fred (1953). Tant qu'il y aura des hommes. Etats-Unis. 